\title{
Tail dependence coefficient of generalized hyperbolic distribution
}

\author{
Mohalilou Aleiyouka \\ Laboratoire de mathématiques appliquées du Havre, Université du Havre Normandie, \\ Le Havre, France \\ mouhaliloune@gmail.com \\ Alexandre Berred \\ Laboratoire de mathématiques appliquées du Havre, Université du Havre Normandie, \\ Le Havre, France \\ alexandre.berred@univ-lehavre.fr \\ Mohammad Ahsanullah \\ Information Systems and Supply Chain Management, Rider University, \\ Lawrenceville, USA \\ ahsan@rider.edu
}

Received 27 June 2016

Accepted 12 July 2017

\begin{abstract}
The tail dependence describes the limiting proportion of exceeding one margin over a certain threshold given that the other margin has already exceeded that threshold. In this paper, we obtain the limit tail dependence coefficient for the generalized hyperbolic distribution.
\end{abstract}

Keywords: Tail dependence; hyperbolic generalized; extreme value.

2000 Mathematics Subject Classification: 62E10, 62E20, 62H05, 62H10, 62H20.

\section{Introduction}

The generalized hyperbolic distribution was introduced in [3], and has been developed by many authors, see among others [17], [12], [16], [2], especially in relation with several applications in the Finance, see [15], [6]. This family contains and generalizes many familiar distributions such as the Student t, Gaussian, variance gamma, Cauchy and others.

The aim of this paper is to investigate the upper tail dependence coefficient in the case of the generalized hyperbolic distribution. More precisely, a bivariate random variable $X=\left(X_{1}, X_{2}\right)$ follows a generalized hyperbolic distribution $G H(\lambda, \alpha, \beta, \delta, \gamma)$ if

$$
X_{1}=\mu_{1}+\theta_{1} W_{1}+\sqrt{W_{1}} Z_{1}, \quad X_{2}=\mu_{2}+\theta_{2} W_{2}+\sqrt{W_{2}} Z_{2},
$$

Copyright (C) 2017, the Authors. Published by Atlantis Press.

This is an open access article under the CC BY-NC license (http://creativecommons.org/licenses/by-nc/4.0/). 
where $Z=\left(Z_{1}, Z_{2}\right) \sim N_{2}(0, \Sigma)$, is the bivariate normal distribution with mean 0 and a correlation matrix $\Sigma$ and $W=\left(W_{1}, W_{2}\right) \sim G I G(\lambda, \delta, \gamma)$, is the generalized inverse gaussian with probability density function

$$
f_{W}(x)= \begin{cases}\frac{\left(\frac{\gamma}{\delta}\right)^{\lambda}}{2 k_{\lambda}(\delta \gamma)} x^{\lambda-1} \exp \left(-\frac{1}{2}\left(\gamma^{2} x+\frac{\delta^{2}}{x}\right)\right. & \text { if } \mathrm{x}>0, \\ 0 & \text { otherwise. }\end{cases}
$$

where $\gamma=\sqrt{\alpha^{2}-\theta^{2}}, \lambda \in \mathbb{R}, \delta>0$ and $\gamma \geq 0$. The probability density function of $G H(\lambda, \alpha, \beta, \delta, \mu)$ is given by

$$
f_{X}(x)=\frac{\left(\alpha^{2}-\theta^{2}\right)^{\frac{\lambda}{2}} k_{\lambda-\frac{1}{2}}\left(\alpha \sqrt{\delta^{2}+(x-\mu)^{2}}\right) \exp (\theta(x-\mu))}{\sqrt{2 \pi} \alpha^{\lambda-\frac{1}{2}} k_{\lambda}\left(\delta \sqrt{\alpha^{2}-\beta^{2}}\right) \delta^{\lambda}\left(\sqrt{\delta^{2}+(x-\mu)^{2}}\right)^{-\lambda+\frac{1}{2}}},
$$

where $K_{\lambda}$ is the modified Bessel function of the third kind, given by

$$
K_{\lambda}(x)=\frac{1}{2} \int_{0}^{\infty} \exp \left(-\frac{x}{2}\left(y+\frac{1}{y}\right)\right) y^{\lambda-1} d u, \quad \text { for } \quad x>0, \lambda \in \mathbb{R},
$$

see for instance, [7], [11]. [1] have given general results for upper tail dependence of skewed grouped $t$-distributions in the case where its degrees of freedom are different, i.e. for a random variable $X=\left(X_{1}, X_{2}\right)$, we can write

$$
X_{1}=\frac{\theta_{1}}{G_{\mu}^{-1}(U) / \mu}+\frac{Z_{1}}{\sqrt{G_{\mu}^{-1}(U) / \mu}}, \quad X_{2}=\frac{\theta_{2}}{G_{v}^{-1}(U) / v}+\frac{Z_{2}}{\sqrt{G_{v}^{-1}(U) / \nu}},
$$

where $Z=\left(Z_{1}, Z_{2}\right)$ is a bivariate normal distribution with mean 0 and correlation matrix $\Sigma=$ $\left(\begin{array}{ll}1 & \rho \\ \rho & 1\end{array}\right)$, with correlation coefficient $\rho$ and $Z$ is independently distributed of the standard uniform random variance $U$. The parameter $\theta=\left(\theta_{1}, \theta_{2}\right)^{\prime} \in \mathbb{R}$ controls the asymmetry of the distribution and $\tau, \nu$ are positive constants. The function $G_{\eta}^{-1}($.$) is the inverse distribution function of \Gamma\left(\frac{\eta}{2}, \frac{\eta}{2}\right)$ with $\eta>0$.

[9] have particularly studied a special case of the skewed grouped $t$-distributions, see [1], when $\eta=v=\tau$ in (1.3), which gives the case of the skew $t$-distribution, introduced in [5]. This model can be defined for $X=\left(X_{1}, X_{2}\right)$ by

$$
X_{1}=\frac{\theta_{1}}{G_{\tau}^{-1}(U)}+\frac{Z_{1}}{\sqrt{G_{\tau}^{-1}(U)}}, \quad X_{2}=\frac{\theta_{2}}{G_{v}^{-1}(U)}+\frac{Z_{2}}{\sqrt{G_{v}^{-1}(U)}},
$$

where $Z_{1}, Z_{2}, \Sigma, \tau$ and $v$ are defined previously.

The coefficient of the lower tail dependence for a bivariate random vector $X=\left(X_{1}, X_{2}\right)$, with marginal distributed functions $F_{1}$ and $F_{2}$ is defined as $\lambda_{L}=\lim _{u \rightarrow 0} \mathbb{P}\left(X_{1} \leq F_{1}^{(-1)}(u) \mid X_{2} \leq F_{2}^{(-1)}(u)\right)$ if this limit exists, where $F_{1}^{-1}$ and $F_{2}^{-1}$ are the generalized inverse functions of $F_{1}$ and $F_{2}$, see [10]. The coefficient of the upper tail dependence of a random vector $X$ can be defined similarly as $\lambda_{U}=\lim _{u \rightarrow 1} \mathbb{P}\left(X_{1} \geq F_{1}^{(-1)}(u) \mid X_{2} \geq F_{2}^{(-1)}(u)\right)$ or equivalently

$$
\lambda_{U}=\lim _{x \rightarrow+\infty} \mathbb{P}\left(X_{1} \geq F_{1}^{(-1)}\left(F_{2}(x)\right) \mid X_{2} \geq x\right) .
$$




\section{Main result}

The aim of this paper is to provide an analytic result of the tail dependence coefficient of the generalised hyperbolic model. We will consider the upper tail dependence for $X$ in the model (1.1), using a method deriving from an equivalent form of (1.4) for $\lambda_{U}$ :

$$
\lambda_{U}=\lim _{x \rightarrow+\infty} \mathbb{P}\left(X_{1} \geq F_{1}^{(-1)}\left(F_{2}(x)\right) \mid X_{2}=x\right)+\lim _{x \rightarrow+\infty} \mathbb{P}\left(X_{2} \geq F_{2}^{(-1)}\left(F_{1}(x) \mid X_{1}=x\right),\right.
$$

see [13].

Theorem 2.1. The upper tail coefficient of the vector $\left(X_{1}, X_{2}\right)$ defined in (1.1) is given by:

1) If $\theta_{1}=\theta_{2}=0$, then

$$
\lambda_{U}=1-\int_{0}^{\infty} g_{\lambda, \gamma, \delta}(t) d t
$$

2) If $\theta_{1}, \theta_{2}>0$, then

$$
\lambda_{U}=1
$$

3) If $\theta_{1}=\theta_{2}=\theta<0$, then

$$
\lambda_{U}=1
$$

Proof. Let $\theta_{1}=\theta_{2}=0$. For $x \rightarrow a$, we use the notation $f(x) \sim g(x)$ if $\frac{f(x)}{g(x)} \rightarrow 1$ and $f(x) \propto g(x)$ if there exists $c>0$, such as $g(x)=c f(x)$. Let $h(x)=F_{1}^{-1} \circ F_{2}(x), g(x)=\sqrt{Q_{\lambda_{1}, \delta_{1}, \gamma_{1}}^{-1} \circ Q_{\lambda_{2}, \delta_{2}, \gamma_{2}}\left(x^{2}\right)}$ and $S^{2}=Q_{\lambda_{2}, \delta_{2}, \gamma_{2}}^{-1}(U)$.

Equation (1.1) can be written as

$$
X_{1}=\mu_{1}+\frac{\theta_{1}}{(g(S))^{2}}+\frac{Z_{1}}{g(S)}, X_{2}=\mu_{2}+\frac{\theta_{2}}{S^{2}}+\frac{Z_{2}}{S},
$$

where $\mu_{1}, \mu_{2}, Z_{1}$ and $Z_{2}$ are defined previously. The upper tail dependence coefficient can be rewritten as

$$
\begin{aligned}
\lambda_{U} & =\lim _{x \rightarrow \infty} \frac{\mathbb{P}\left(\left(X_{1} \geq h(x), X_{2} \geq x\right)\right.}{\mathbb{P}\left(X_{2} \geq x\right)}, \\
& =\lim _{x \rightarrow \infty} \frac{\int_{h(x)}^{\infty} \int_{x}^{\infty} f_{X_{1}, X_{2}}(s, t) d s d t}{\mathbb{P}\left(X_{2} \geq x\right)},
\end{aligned}
$$

We use the l'Hopital's rule to obtain

$$
\begin{aligned}
\lambda_{U} & =\lim _{x \rightarrow \infty} \frac{-\int_{h(x)}^{\infty} f_{X_{1}, X_{2}}(s, x) d s-h^{\prime}(x) \int_{x}^{\infty} f_{X_{1}, X_{2}}(h(x), t) d t}{-f_{2}(x)}, \\
& =\lim _{x \rightarrow \infty}\left(\mathbb{P}\left(X_{1} \geq h(x) / X_{2}=x\right)+h^{\prime}(x) \frac{f_{1}(x)}{f_{2}(x)} \mathbb{P}\left(X_{2} \geq x / X_{1}=h(x)\right)\right) .
\end{aligned}
$$

The distribution function $Q_{W_{2}}$ of the generalized inverse gaussian is given by

$$
Q_{W_{2}}(x)=\int_{\infty}^{x} \frac{\left(\frac{\gamma}{\delta}\right)^{\lambda}}{2 k_{\lambda}(\delta \lambda)} t^{\lambda-1} \exp \left(-\frac{1}{2}\left(\gamma^{2} t-\frac{\delta^{2}}{t}\right) d t .\right.
$$


It follows that the corresponding distribution function $Q_{W_{2}}$ satisfies, as $x \rightarrow \infty, 1-Q_{W_{2}}(x) \sim$ $c_{\lambda, \gamma, \delta} \frac{x^{\lambda}}{\lambda} \exp \left(-\frac{1}{2} \gamma^{2} x\right)$, where $c_{\lambda, \gamma, \delta}=\frac{\left(\frac{\gamma}{\delta}\right)^{\lambda}}{2 k_{\lambda}(\delta \lambda)}$. Then

$$
Q_{W_{2}}(x)=1-c_{\gamma, \delta} \frac{x^{\lambda}}{\lambda} \cdot \exp \left(-\frac{1}{2} \gamma^{2} x\right)
$$

We use Lemma 3.1, see [1], to invert the last equation and obtain, as $u \rightarrow 1$,

$$
Q_{W_{2}}^{-1}(u)=-\frac{\log (1-u)}{\gamma^{2} / 2} \text {. }
$$

Hence for $x \rightarrow \infty$

$$
g(x)=m_{\gamma_{2}, \delta_{2}} x^{2 \lambda_{2}} \exp \left(-\frac{1}{2} \gamma_{2}^{2} x^{2}\right), \quad \text { where } \quad m_{\lambda_{2}, \gamma_{2}, \delta_{2}}=2 \frac{c \lambda_{2}, \gamma_{2}, \delta_{2}}{\lambda_{2} \gamma_{2}^{2}} .
$$

The density $f_{1}$ of the generalized hyperbolic distribution with parameters $\lambda, \alpha, \theta, \delta, \gamma$ is given by

$$
f_{1}(x)=\ell_{\lambda}(\alpha, \theta, \delta) k_{\lambda-\frac{1}{2}}\left(\alpha \sqrt{\delta^{2}+(x-\mu)^{2}}\right)\left(\sqrt{\delta^{2}+(x-\mu)^{2}}\right)^{\lambda-\frac{1}{2}} \exp (\theta(x-\mu)),
$$

where $\ell_{\lambda}(\alpha, \theta, \delta)=\frac{\left(\alpha^{2}-\theta^{2}\right)^{\frac{\lambda}{2}}}{\sqrt{2 \pi} \alpha^{\lambda-\frac{1}{2}} \delta^{\lambda} k_{\lambda}\left(\delta \sqrt{\alpha^{2}-\beta^{2}}\right)}$. It follows that the corresponding distribution function $F_{1}$ satisfies, as $x \rightarrow \infty$,

$$
\begin{aligned}
1-F_{1}(x) & \sim \int_{x}^{\infty} \ell_{\lambda}(\alpha, \theta, \delta) t^{\lambda-1 / 2} k_{\lambda-\frac{1}{2}}\left(\alpha \sqrt{\delta^{2}+(t-\mu)^{2}}\right) \exp (\theta(t-\mu)) d t, \\
& \sim d_{\lambda} x^{\lambda} \exp (-(\alpha-\theta) x),
\end{aligned}
$$

where $d_{\lambda}=\frac{\pi}{2 \lambda \alpha} \ell_{\lambda}(\alpha, \theta, \delta)$. Using the same lemma as above, for $u \rightarrow 1$, we can invert $F_{1}$ and obtain $F_{1}^{-1}(u)=-\frac{\log (1-u)}{\alpha-\theta}$. It follows that the strictly increasing function $h(x)=F_{1}^{-1} \circ F_{2}(x)$ satisfies, as $x \rightarrow \infty$,

$$
h(x) \sim(\alpha-\theta) x
$$

The derivative of $h$ is equal to $h^{\prime}=\frac{f_{2}}{f_{1} \circ h}$, and hence, as $x \rightarrow \infty$,

$$
\frac{f_{1}(x)}{f_{2}(x)} h^{\prime}(x)=\frac{f_{1}(x)}{f_{1} \circ h(x)} \sim(\alpha-\theta)^{1-\lambda} \exp (-(\alpha-\theta) x(1-(\alpha-\theta)) .
$$

Note that this quantity tends to zero for $\alpha<1+\theta$. Hence the second term in (2.1) goes to zero. It remains to calculate the first term of the equation (2.1). The conditional density of $S^{2}$ given $X_{2}$ satisfies, by Bayes rule,

$$
\begin{aligned}
p_{S^{2} \mid X_{2}=x_{2}}(t) & \propto p_{X_{2} \mid S^{2}=t}\left(x_{2}\right) p_{S^{2}}(t), \\
& \propto t^{(\lambda-1 / 2)-1} \exp \left(-\frac{1}{2}\left(\gamma^{2} t+\frac{\delta^{2}+x^{2}}{t}\right) .\right.
\end{aligned}
$$

Then $S^{2} \mid X_{2}=x_{2} \sim G I G\left(\lambda-1 / 2, \gamma, \sqrt{\delta^{2}+x^{2}}\right)$. Now, we compute the second term of (2.1).

$$
\begin{aligned}
\mathbb{P}\left(X_{1} \geq x_{1} \mid X_{2}=x_{2}\right) & =\int_{0}^{\infty} \mathbb{P}\left(X_{1} \geq x_{1} \mid X_{2}=x_{2}, s^{2}=t\right) f_{S^{2} \mid X_{2}=x_{2}}(t) d t \\
\geq & =\int_{0}^{\infty} \mathbb{P}\left(\frac{1}{g(\sqrt{t})}\left(\rho \sqrt{t} x_{2}+\left(1-\rho^{2}\right) W\right) \geq x_{1}\right) g_{\lambda^{\prime}, \gamma, \delta^{\prime}}(t) d t
\end{aligned}
$$


where $\lambda^{\prime}=\lambda-1 / 2, \delta^{\prime}=\sqrt{\delta^{2}+x^{2}}$ and $W \sim \mathscr{N}(0,1)$. We can re-express the right side of (2.1) as $\lim _{x \rightarrow \infty} \int_{0}^{\infty} \mathbb{P}\left(\left(1-\rho^{2}\right) W \geq f(x) g(t)-\rho \sqrt{t} x\right) g_{\lambda^{\prime}, \gamma, \delta^{\prime}}(t) d t$. Using the dominated convergence theorem, we obtain $\lambda_{U}=1-\int_{0}^{\infty} g_{\lambda^{\prime}, \gamma, \delta^{\prime}}(t) d t$.

Let $\theta_{1}, \theta_{2}>0$ or $\theta_{1}, \theta_{2}<0$, For our quantitative developement we need the modified Bessel function of the third kind, see [14], with index $\lambda \in \mathbb{R}$ :

$$
K_{\lambda}(x)=\sqrt{\frac{\pi}{2 x}} \exp (-x)\left(1+o\left(\frac{1}{x}\right)\right)
$$

According to the equation (1.2), we have

$$
\begin{gathered}
\frac{K_{\lambda+1}(x)}{K_{\lambda}(x)}=1+\frac{8 \lambda+4}{8 x}+\frac{32 \lambda^{2}-8}{(8 x)^{2}}+o\left(\frac{1}{x^{3}}\right), \\
\frac{K_{\lambda+1}(x) K_{\lambda-1}(x)}{\left(K_{\lambda}(x)\right)^{2}}=1+\frac{1}{x}+\frac{64-256 \lambda^{2}}{(8 x)^{3}}+o\left(\frac{1}{x^{4}}\right) .
\end{gathered}
$$

The marginal density of $X_{1}$ is defined by

$$
f_{X_{1}}\left(x_{1}\right)=\frac{k_{\lambda-\frac{1}{2}}\left(\delta^{2}+\left(x_{1}-\mu_{1}\right)^{2}, \alpha^{2}\right)}{\sqrt{2 \pi} k_{\lambda}\left(\delta^{2}, \alpha^{2}-\theta_{1}^{2}\right)} e^{\theta_{1}\left(x_{1}-\mu_{1}\right)},
$$

where $K_{\lambda}\left(\delta^{2}, \alpha^{2}-\beta^{2}\right)=\int_{0}^{\infty} x^{\lambda-1} e^{-\frac{1}{2}\left(\left(\alpha^{2}-\beta^{2}\right) x+\frac{\delta^{2}}{x}\right)} d x$. The conditional density of $X_{2.1}=X_{2} \mid X_{1}=x_{1}$ is equal to

$$
\begin{aligned}
f_{X_{2} \mid X_{1}}\left(x_{2} \mid x_{1}\right)= & \frac{f_{X_{1}, X_{2}}\left(x_{1}, x_{2}\right)}{f_{X_{1}}\left(x_{1}\right)} \\
& =\frac{k_{\lambda-1}\left(\delta^{2}+a\left(x_{1}, x_{2}\right), \alpha^{2}\right)}{\sqrt{2 \pi} k_{\lambda-\frac{1}{2}}\left(\delta^{2}+\left(x_{1}-\mu_{1}\right)^{2}, \alpha^{2}\right)} e^{\theta_{2}\left(x_{2}-\mu_{2}\right)},
\end{aligned}
$$

where $a\left(x_{1}, x_{2}\right)=\frac{1}{\left(1-\rho^{2}\right)}\left(\left(x_{1}-\mu_{1}\right)^{2}+2\left(x_{1}-\mu_{1}\right)\left(x_{2}-\mu_{2}\right)+\left(x_{2}-\mu_{2}\right)^{2}\right)$. Note that $X_{2.1}$ has a generalised hyperbolic distribution. It is known that the conditional distribution of $X_{2.1} \mid W=w$ is normal $N\left(\mu_{2}+\theta_{2} w, w\right)$, where $W \sim G I G\left(\lambda-\frac{1}{2}, \delta^{2}+\left(x_{1}+\mu_{1}\right)^{2}, \alpha^{2}+\theta_{2}^{2}\right)$.

The proof we propose here requires the use of the Chebyshev's inequality, so we compute the first two moments. We have

$$
\begin{aligned}
\mathbb{E}\left(X_{2.1}\right) & =\mu+\theta_{2} \mathbb{E}(W) \\
& =\mu+\theta_{2} \frac{\delta^{2}+\left(x_{1}+\mu_{1}\right)^{2}}{\alpha^{2}+\theta_{2}^{2}} \frac{K_{\lambda+\frac{1}{2}}\left(\left(\alpha^{2}+\theta_{2}^{2}\right)\left(\delta^{2}+\left(x_{1}+\mu_{1}\right)^{2}\right)\right.}{K_{\lambda-\frac{1}{2}}\left(\left(\alpha^{2}+\theta_{2}^{2}\right)\left(\delta^{2}+\left(x_{1}+\mu_{1}\right)^{2}\right)\right.},
\end{aligned}
$$

You can easily compute the variance of $X_{2.1}$ from the variance decomposition formula and obtain

$$
\begin{aligned}
\operatorname{Var}\left(X_{2.1}\right)= & \theta_{2}^{2}\left(\frac{\delta^{2}+\left(x_{1}+\mu_{1}\right)^{2}}{\alpha^{2}+\theta_{2}^{2}}\right)^{2}\left[\frac{K_{\lambda+\frac{1}{2}}\left(\left(\alpha^{2}+\theta_{2}^{2}\right)\left(\delta^{2}+\left(x_{1}+\mu_{1}\right)^{2}\right)\right.}{K_{\lambda-\frac{1}{2}}\left(\left(\alpha^{2}+\theta_{2}^{2}\right)\left(\delta^{2}+\left(x_{1}+\mu_{1}\right)^{2}\right)\right.}\right]^{2} \\
& \times\left(\frac{K_{\lambda+\frac{3}{2}}\left(\left(\alpha^{2}+\theta_{2}^{2}\right)\left(\delta^{2}+\left(x_{1}+\mu_{1}\right)^{2}\right)\right.}{K_{\lambda-\frac{1}{2}}\left(\left(\alpha^{2}+\theta_{2}^{2}\right)\left(\delta^{2}+\left(x_{1}+\mu_{1}\right)^{2}\right)\right.}-1\right) \\
& +\theta_{2} \frac{\delta^{2}+\left(x_{1}+\mu_{1}\right)^{2}}{\alpha^{2}+\theta_{2}^{2}} \frac{K_{\lambda+\frac{1}{2}}\left(\left(\alpha^{2}+\theta_{2}^{2}\right)\left(\delta^{2}+\left(x_{1}+\mu_{1}\right)^{2}\right)\right.}{K_{\lambda-\frac{1}{2}}\left(\left(\alpha^{2}+\theta_{2}^{2}\right)\left(\delta^{2}+\left(x_{1}+\mu_{1}\right)^{2}\right)\right.}
\end{aligned}
$$


By the Chebyshev's Inequality, for all positive $\varepsilon$,

$$
\begin{aligned}
\mathbb{P}\left(\left|X_{2.1}-\mathbb{E}\left(X_{2.1}\right)\right| \geq \varepsilon\left|x_{1}\right|\right) & \leq \frac{\operatorname{Var}\left(X_{2.1}\right)}{\varepsilon^{2}\left|x_{1}\right|^{2}} \\
& =\frac{1}{\varepsilon^{2}\left|x_{1}\right|^{2}}\left[\left(\delta^{2}+\left(x_{1}-\mu_{1}\right)^{2}\right)\left(\frac{\alpha^{2}+2 \theta_{2}^{2}}{\left(\alpha^{2}+\theta_{2}^{2}\right)^{3}}\right)\right. \\
& \left.+\lambda\left(\frac{\alpha^{2}+3 \theta_{2}^{4}}{\left(\alpha^{2}+\theta_{2}^{2}\right)^{4}}\right)+o\left(\frac{1}{\left|x_{1}\right|}\right)\right]=o\left(\frac{1}{\left|x_{1}\right|}\right) .
\end{aligned}
$$

We will combine the variance of $X_{2.1}$ with (2.3) and (2.4), as $x_{1} \rightarrow-\infty$. This gives

$$
\mathbb{P}\left(\mathbb{E}\left(X_{2.1}\right)-\varepsilon\left|x_{1}\right| \leq X_{2.1} \leq \mathbb{E}\left(X_{2.1}\right)+\varepsilon\left|x_{1}\right|\right) \geq 1-o\left(\frac{1}{\left|x_{1}\right|}\right) \text {, as } x_{1} \rightarrow-\infty,
$$

Hence $\lim _{x_{1} \rightarrow-\infty} \mathbb{P}\left(X_{2} \leq F_{2}^{-1}\left(F_{1}(y)\right) \mid X_{1}=x_{1}\right)=0$ if

$$
\begin{aligned}
F_{2}^{-1}\left(F_{1}\left(x_{1}\right)\right) & <\mathbb{E}\left(X_{2.1}\right)-\varepsilon\left|x_{1}\right| \\
& =\left(\mu_{2}-\frac{\theta_{2}}{\left(\alpha^{2}+2 \theta_{2}^{2}\right)^{2}}+\varepsilon\right) x_{1}+\lambda \frac{\theta_{2}}{\left(\alpha^{2}+2 \theta_{2}^{2}\right)^{2}}+o\left(\frac{1}{\left|x_{1}\right|}\right),
\end{aligned}
$$

We use the expectation of the random variable $X_{2.1}$ and (2.3), as $x_{1} \rightarrow-\infty$, to show

$$
F_{1}\left(x_{1}\right)<F_{2}\left(\left(\mu_{2}-\frac{\theta_{2}}{\left(\alpha^{2}+2 \theta_{2}^{2}\right)^{2}}+\varepsilon\right) x_{1}+\lambda \frac{\theta_{2}}{\left(\alpha^{2}+2 \theta_{2}^{2}\right)^{2}}+o\left(\frac{1}{\left|x_{1}\right|}\right)\right) .
$$

Hence for $i=1,2$,

$$
\begin{aligned}
F_{i}\left(x_{1}\right) & =\mathbb{P}\left(X_{i} \leq x_{1}\right) \\
& =\frac{x^{\lambda-\frac{3}{2}} e^{\left(\theta_{i}^{2}+\alpha^{2}+\theta_{i}\right) x_{1}}}{2 K_{\lambda}\left(\delta^{2}, \alpha^{2}-\theta_{1}^{2}\right)\left(\theta_{i}^{2}+\alpha^{2}\right)^{(\lambda-1 / 2) / 2}\left(\theta_{i}^{2}+\alpha^{2}+\theta_{i}\right)}\left(1+o\left(\frac{1}{\left|x_{1}\right|}\right)\right) .
\end{aligned}
$$

By combining (2.7) and (2.6), we have

$$
\left(\theta_{1}^{2}+\alpha^{2}+\theta_{1}\right)>\left(\theta_{2}^{2}+\alpha^{2}+\theta_{2}\right)\left(\mu_{2}-\frac{\theta_{2}}{\sqrt{\theta_{1}^{2}+b^{2}}}+\varepsilon\right) .
$$

as $\varepsilon>0$ is arbitrary, we obtain $\frac{\theta_{1}^{2}+\alpha^{2}+\theta_{1}}{\theta_{2}^{2}+\alpha^{2}+\theta_{2}}-\left(\mu_{2}-\frac{\theta_{2}}{\left(\alpha^{2}+2 \theta_{2}^{2}\right)^{2}}\right)>0$. If $\theta_{1}=\theta_{2}=\theta \in \mathbb{R}^{*}$, then $1-$ $\mu_{2}+\frac{\theta}{\left(\alpha^{2}+2 \theta^{2}\right)^{2}}>0$. Then $\lim _{x_{1} \rightarrow+\infty} \mathbb{P}\left(X_{2} \geq F_{2}^{-1}\left(F_{1}(y)\right) \mid X_{1}=x_{1}\right)=1$. Similarly, one can show that $\lim _{x_{1} \rightarrow+\infty} \mathbb{P}\left(X_{1} \geq F_{1}^{-1}\left(F_{2}(y)\right) \mid X_{2}=x_{1}\right)=0$ and results in $\lambda_{U}=1$.

\section{References}

[1] K. Banachewicz and A. van der Vaart, Tail dependence of skewed grouped $t$-distributions. Statistics and Probability Letters. 78 (2008) 2388-2399.

[2] O. E. Barndorff-Nielsen, and H. Christian, Infinite divisibility of the hyperbolic and generalized inverse Gaussian distributions. Probability Theory and Related Fields, Springer. 38(4) (1977) 309-311.

[3] O. E. Barndorff-Nielsen, Exponentially decreasing distributions for the logarithm of particle size, Royal Society of London. Proceedings A. Mathematical, Physical and Engineering Sciences. 353 (1977) 401419.

[4] J. Beirlant, Y. Goegebeur, J. Teugels and J. Segers, Statistics of Extremes: Theory and Applications, Wiley, Chichester. (2006). 
[5] S. Demarta, A. J. McNeil, The $t$ copula and related copulas, International Statistical Review. 73 (2005) 111-129.

[6] E. Eberlein and K. Prause, The generalized hyperbolic model: Financial derivatives and risk measures, In Mathematical Finance-Bachelier Congress 2000, H. German, D. Madan, S. Pliska and T. Vorst (Eds). (2002) 245-267.

[7] A. Erdelyi,W. Magnus, F. Oberhettinger and F. G. Tricomi, Bateman Manuscript Project: Tables of Integral Transform, McGraw-Hill, New York. (1954).

[8] E. Jondeau, Asymmetry in tail dependence in equity portfolios.Computational Statistics \& Data Analysis (2008).

[9] T. Fung and E. Seneta, Tail dependence for two skew t distributions, Statistics and Probability Letters. 80 (2010) 784-791.

[10] H. Joe, Multivariate Models and Dependence Concepts, Chapmann \& Hall, London. (1997).

[11] B. Jorgensen, Statistical properties of the generalized inverse Gaussian distribution for log-linear and stochastic volatility models, Lecture Notes in Statistics. (1982).

[12] A.J. McNeil, R. Frey and P. Embrechts, Quantitative Risk Management: Concepts, Techniques and Tools, Princeton University Press. (2005).

[13] R. B. Nelsen, An Introduction to Copulas, Lecture Notes in Statistics, vol. 139. (1999).

[14] M. Paolella, Intermediate Probability: A Computational Approach, Wiley, Chichester. (2007).

[15] K. Prause, The Generalized Hyperbolic Model: Estimation Financial Derivatives and Risk Measures, PhD diss., University of Freiburg. (1999).

[16] R. Schmidt, Tail dependence for elliptically contoured distributions, Mathematical Methods of Operations Research. 55 (2002) 301-327.

[17] S. Schlueter and M. Fischer, The weak tail dependence coefficient of the elliptical generalized hyperbolic distribution, Extremes. 15(2) (2012) 159-174. 